\section{Are GM Crops for Yield and Resilience Possible?}

\author{
Matthew J. Paul, ${ }^{1, \star}$ Michael L. Nuccio, ${ }^{2}$ and \\ Shib Sankar Basu ${ }^{2,3}$
}

Crop yield improvements need to accelerate to avoid future food insecurity. Outside Europe, genetically modified (GM) crops for herbicide- and insectresistance have been transformative in agriculture; other traits have also come to market. However, GM of yield potential and stress resilience has yet to impact on food security. Genes have been identified for yield such as grain number, size, leaf growth, resource allocation, and signaling for drought tolerance, but there is only one commercialized drought-tolerant GM variety. For GM and genome editing to impact on yield and resilience there is a need to understand yield-determining processes in a cell and developmental context combined with evaluation in the grower environment. We highlight a sugar signaling mechanism as a paradigm for this approach.

\section{Transformative GM Traits}

GM of plants through Agrobacterium-mediated plant transformation was first reported in 1982 [1,2]. The first GM crops followed in 1996, increasing acreage >100-fold by 2015, from 1.7 million to 180 million hectares. This was the fastest adopted crop technology in recent history, and was in the same class as the Green Revolution of the 1960s. According to a report from the International Service for the Acquisition of Agri-Biotech Applications (ISAAA) in 2015,' the percentage GM areas planted for the four principal GM crops worldwide soybean, cotton, maize, and canola - are $83 \%, 78 \%, 29 \%$, and $24 \%$, respectively. These GM crops have been dominated by herbicide- and insect/pest-resistant traits. The largest review yet conducted concluded that the effects of GM crops on farming were overwhelmingly positive [3]. Herbicide-tolerant crops have lower production costs by transforming weed control and enabling no-till production. For insect-resistant crops expressing Bacillus thuringiensis, Bt, it is estimated that between 1996 and 2011 the total global consumption of pesticides decreased by 9\% [4], saving farmers $\$ 31$ billion and $\$ 26$ billion on pesticides for cotton and maize, respectively. Higher seed prices were offset by the benefits of growing these crops, leaving overall production costs about the same. Yields increased $9 \%$ for herbicide-tolerant crops and $25 \%$ for insect-resistant crops. About $65 \%$ of the gains have been from increased yield, with $35 \%$ of the gains being from cost savings. Farmers who adopted GM crops made 69\% higher profits than those who did not. In developing countries GM crops have increased yields by $14 \%$ on average. These first GM input traits made crop production easier and more efficient for the farmer while at the same time reducing yield losses. GM traits in maize, soybean, and cotton contribute more than $\$ 160$ billion per year [4]. The technology has been transformational.

Other traits that have been developed have been disease resistance, quality and nutritional enhancement traits, improvement of shelf life, and modification of crops for non-food uses. A non-exhaustive list includes papaya resistant to ringspot virus among the first successfully commercialized GM crops [5]. Quality and nutritional traits include Flavr Savr
Trends

GM by foreign gene insertion for herbicide- and insect-resistance traits has been transformative in soybean, cotton, maize, and canola. GM has made crop production easier and more efficient for the farmer, reducing yield losses, costs, and chemical inputs, thereby increasing productivity and farmer profitability.

GM of yield potential and yield resilience, however, despite showing promise in some cases in the laboratory, have yet to impact on agriculture based on major crops. There is only one example of a commercialized drought-resilient GM crop

Recently, a sugar signaling mechanism regulated by trehalose 6-phosphate, and targeted by a trehalose phosphate phosphatase in a cell and developmental manner, gave large increases in yield in the food security crop maize in extensive field trials with and without drought during the flowering period.

${ }^{1}$ Plant Biology and Crop Science, Rothamsted Research, Harpenden, Hertfordshire, AL5 2JQ, UK

2Syngenta Crop Protection, LLC, Research Triangle Park, NC, USA ${ }^{3}$ Current address: Symmetry Bioanalytics LLC, Research Triangle Park, NC, USA

*Correspondence: matthew.paul@rothamsted.ac.uk (M.J. Paul). 
tomato, golden vitamin A rice, high-lysine corn, canola, and soy, and high-iron rice [6]. Not all, for example golden rice, have yet come to market. RNAi suppression of asparagine synthase in Innate ${ }^{\mathbb{R}}$ potato lowers asparagine levels, a precursor of acrylamide, a carcinogen that can form when potatoes are fried [7]. Corn expressing a thermally stable amylase Enogen $^{\mathrm{TM}}$ which increases the efficiency of starch breakdown $[8,9]$ is used in bioethanol production. Other non-food uses include alteration of lignin for paper pulping and feedstock digestibility [10,11], production of replacements for plastics [12], and molecular pharming [13].

These GM traits were enabled by transgenesis of genes that did not engage with or perturb the mainstream growth processes or resource acquisition and allocation of the plants. Successful traits typically could be enabled with a single dominant transgene requiring ubiquitous expression. Even though this technology needed careful development, it can be considered nowadays to be relatively straightforward.

\section{GM of Yield}

At the same time as these GM success stories, GM of intrinsic plant processes that determine yield, which are naturally more complex and sophisticated, was also carried out. Targeting any intrinsic plant process to improve productivity or abiotic stress resilience, however, has not yet produced breakthroughs for major food security crops in agriculture. Other than the often-complex issues of introducing new GM crops, there are biological reasons for lack of development of GM crops for higher yield. A main explanation is the complex cell- and developmental-specific, as well as multigenic, nature of yield control which has not been amenable to improvement with single genes controlled by constitutive promoters. Second, single genes, unless they are key regulatory genes, are then buffered or counteracted by the strong homeostatic mechanisms of growth control of the plant. There have been many reports of transgenic plants targeting metabolism that perform well in laboratory or greenhouse conditions, such that by 2000 there were many field trial applications for GM traits designed to increase yield [14]. More recently overexpression of cell wall invertase in tomato improved yield under salinity [15], demonstrating that sucrose utilization may be a good target. Overexpression of ADP-glucose pyrophosphorylase, the key enzyme of starch synthesis, in wheat grain improved wheat yields substantially [16]. In China, indirect selection over 100 years of sucrose synthase, which breaks down sucrose for delivery into starch synthesis, confirms delivery of sucrose for starch synthesis as a major target for wheat yield improvement [17]. Expression of a barley sucrose transporter in wheat appears to improve yield in the field [18]. Other ways to improve source and sink strength may be through modifying hormone signaling [19]. Some good examples include modification of auxin signaling to increase yield [20] and grain size [21]. Sedoheptulose-1,7bisphosphatase (SBPase) has been highlighted as a promising candidate in the improvement of photosynthesis and yield [22]. Most dominant single-gene traits have been largely optimized by breeders. It would be remarkable perhaps if the overexpression of a single photosynthesis gene improved crop yield, and this raises interesting questions about why breeding for yield has not done this already. Linkage drag and lack of recombination could be possible reasons, or because in a field canopy over the full course of a season improving photosynthesis does not noticeably increase yield, and constraints remain elsewhere. Perhaps the best example of a GM crop improved for yield is in Eucalyptus. Overexpression of an $A$. thaliana endo-1,4- $\beta$-glucanase (ce/1) driven by its own promoter and expressing specifically in elongation zones induces rapid elongation of cells through the hydrolysis of cellulose-xyloglucan links [23]. This results in faster growth such that trees can be harvested in 5.5 rather than 7 years, and with $20 \%$ more wood, and is one of very few examples where targeting a metabolic enzyme benefits growth and yield. GM Eucalyptus was approved for cultivation in Brazil in 2015. 
As far as we know, apart from trials including the genes and traits for yield enhancement in the crops mentioned above $[18,23]$ have either been unsuccessful or have not been carried out, and consequently have not come to market. To evaluate the efficacy and robustness of any of these promising traits, field trials need to be conducted using parameters that growers typically use. The environmental variability and heterogeneity of field conditions are unique to the field environment and cannot be replicated in a controlled environment. Welldesigned productivity trials in grower environments are required. Promising transgenes in the laboratory may encounter unknown and potentially unknowable variables in the field. Hence new varieties must be tested at multiple sites in genetic backgrounds suited to the site, and in different years, to address environmental variability between seasons. Standard practice protocols for the testing of GM crops have been published [24].i. Important considerations of penetrance and heritability should be considered. Penetrance, in other words the ability of a genetic modification to achieve a consistent outcome among different elite cultivars in addition to heritability, is crucial for consistent yield performance and commercialization of GM traits. Simple genetic tests of penetrance and heritability can determine whether a GM trait is worth further investment.

Further examples that may start to impact on crop yields outside direct effects on metabolism where yield has been improved in the field are in soybean, maize, and rice. In transgenic soybean, large-scale screening for yield identified an $A$. thaliana B-box domain gene (AtBBX32) which may modulate the circadian clock to increase the duration of reproductive development [25]. Constitutive expression of this transcription factor increased plant height as well as pod and total seed number, with an up to $14 \%$ increase in total yield. Similar largescale screening of candidate genes in transgenic corn identified another $A$. thaliana transcription factor, a member of homeodomain leucine zipper II (HD-Zip II) family, HB17 [26]. AtHB17 under the control of a constitutive promoter produced a truncated protein that was proposed to weaken transcriptional repression activity of endogenous corn HD-Zip II proteins through protein interactions. Consequently, depending on the environmental conditions, sink potential is enhanced via increased kernel weight or number [27]. Another example is overexpression of microRNA OsmirR397 which increased rice seed size and yield in field trials through modification of brassinosteroid synthesis [28]. The regulation of stem cell proliferation in maize reproductive structures is also showing promise [29]. It is notable that all these examples as well as those for metabolism increase yield by modifying growth and sink strength.

\section{GM of Drought Tolerance}

Drought is the most widespread abiotic stress with the largest impact on crop yield worldwide, and hence is an important crop improvement target. There are numerous examples of engineering drought tolerance that appear to be effective in the laboratory. However, many of these examples slow growth, meaning that less water is lost but this is at the expense of productivity [30]. This is not an acceptable trade-off for growers.

There are examples of some potential leads for drought tolerance involved in signal transduction pathways or transcription factors, for example DREBs involved in ABA signaling [6]. Targeting ethylene signaling looks particularly promising. Overexpression of ARGOS1 is thought to desensitize the plant to ethylene [31,32], and using the maize ubiquitin promoter improved maize yields in some drought environments [33]. Recently the use of CRISPR/Cas9 to target ARGOS8 in maize increased yield in the field [34]. RNAi gene suppression to downregulate 1-aminocyclopropane-1-carboxylic acid synthase, the rate-limiting step of ethylene biosynthesis, has also been successful [35]. 
The only commercially available drought-tolerant crop overexpresses the Bacillis subtilis cold shock protein $\mathrm{B}(\mathrm{CspB}$ ) in maize under the control of rice actin (Ract1) promoter. This improves plant performance under drought imposed during vegetative as well as reproductive development. The nucleic acid-binding ability of this bacterial RNA chaperone is essential for the observed drought tolerance [36]. CspB gene expression stabilizes plant RNA and helps plant cells to produce proteins that are essential for growth which supports yield formation when water is scarce. Transgenic plants produced more chlorophyll and had higher photosynthetic rates. However, the precise mechanistic basis by which CspB mediates plant RNA stabilization and positively influences plant performance during drought is not fully understood. The commercially available CspB maize trait improved grain yield by $6 \%$ when water-deficit was imposed at mid-vegetative to mid-reproductive stages in multi-year field testing [37].

\section{Drought Tolerance in a Developmental Context}

Regarding GM, drought has seldom been approached in a cell- or developmental-specific way, often addressing symptoms of drought rather than underlying causes of yield loss [38,39]. In cereals the flowering period is particularly sensitive to water deficit. Drought during early reproductive development reduces grain set, and this cannot be recovered later except by increasing the size of remaining grain, but this does not fully compensate. During drought sucrose accumulates in vegetative tissues, and the flow of sucrose or use of sucrose in developing reproductive structures appears to be limited [40-42]. Hence maintaining or enhancing sucrose flow would be a potential means of improving performance under drought, in other ways through improving carbon allocation to harvested parts of the plant, grain, seed, or tubers.

Combining Yield Potential and Resilience by Targeting Source-Sink Balance Albecete et al. [15] proposed the benefits of optimizing source-sink balance for better performance under abiotic stresses. A recent publication in Nature Biotechnology [42] reporting field trials of higher yielding GM maize targeting trehalose metabolism vindicates this approach for delivery under field conditions. In 1997 and 2001 Goddijn and coworkers [43,44] highlighted a sugar signaling mechanism that offered promise for rational GM of photosynthesis and yield linked to growth and development through targeting the regulation of metabolism and, in particular, the emerging area of trehalose signaling. Nuccio et al. [42] demonstrate that, if seed set can be preserved and improved during the crucial early flowering period, then yield can be increased under a range of water supplies from no drought to severe drought during the flowering period. Yield was increased up to $10 \%$ without drought and up to $120 \%$ under severe drought. This also occurred without compromising seed size. Interestingly, improved performance under limiting water availability is through altered carbon allocation rather than through improved water use efficiency.

The OsMADS6 promoter used by Nuccio et al. [42] has a cell and developmental expression profile during the flowering period in vascular tissue and developing kernels [24,42,45]. When linked to a rice trehalose phosphate phosphatase (TPP) gene the sucrose content of developing kernels is increased [42]. Use of this construct may be a way to increase yield potential and sink strength in maize. It is thought that this has been achieved by driving down levels of T6P and breaking the sucrose:T6P nexus which may be strongly regulated to maintain sucrose homeostasis [46]. T6P may be part of a mechanism to keep sucrose levels within a specific range for some tissues and cells. Drawing T6P down to an artificially lower level in specific cells or tissues may create a starvation signal for import of more sucrose into developing kernels. This may be a way to increase kernel set, seed number, and yield. T6P regulation of SnRK1 provides a potential mechanism for this $[42,47]$. Both T6P and SnRK1 are associated with changes in whole-plant carbon allocation $[48,49]$, and could indeed operate together through T6P regulation of SnRK1, with T6P providing the sugar availability input into this carbon and energy sensor [47] (Box 1). 
Box 1. Trehalose 6-Phosphate (T6P)

T6P is a signal of sucrose availability in plants. Targeting T6P in a cell and developmental manner has provided one of the few examples of yield enhancement by GM for a major crop after extensive field trialing [42]. Through inhibition of the feast famine protein kinase, SnRK1 [47], T6P causes metabolic reprogramming through changes in gene expression in favor of growth and development and the synthesis of end-products such as starch. When T6P is in low abundance under low assimilate supply a different set of genes are activated that are involved in mobilizing storage reserves, sucrose transport, and other stress and survival responses. Decreasing T6P in maize reproductive structures over the 2 week flowering period alters sucrose allocation in favor of kernels, thereby preventing kernel abortion under drought [42]. Decreasing T6P in germinating rice enables more effective mobilization of starch reserves to enable germination under flooding-induced anaerobic conditions, meaning that rice can be direct seeded, thus removing the need for transplanting and associated labor requirement and yield losses [51]. Increasing T6P in grain during grain filling increases starch synthesis and grain size, whereas increasing T6P in vegetative tissue before rewatering after drought improves growth recovery from drought [50].

\section{Concluding Remarks and Future Perspectives}

The yield gains in the field reported in Nuccio et al. [42] are the type of breakthrough that was once expected routinely for yield by GM. Given that yield is a multigenic trait with low heritability, the specific expression of only one gene to improve it is remarkable. This is because T6P has been carefully targeted by using the MADS6 promoter and because T6P is a master regulator of scores of genes which may be central to source-sink balance. It demonstrates that sucrose flow to sinks is not yet optimized in crops. Recently, chemical modification of T6P levels in wheat grain increased grain size by up to $20 \%$ [50]. Further, Kretzschmar et al. [51] identified a TPP underpinning a QTL for enhanced germination under flooding. All three examples (GM, chemical intervention, QTL) emphasize the utility of targeting T6P for yield improvement in different conditions. Since 1997 the field of trehalose signaling has transitioned from curiosity interest to mainstream plant and crop science aimed at making the regulation of sucrose use and allocation amenable for crop yield improvement. Fortunately, in the case of trehalose signaling fundamental science has run alongside field evaluations to deliver yield improvements with a strong element of understanding the mechanisms and their developmental- and cell-specific contexts $[42,47]$. The research strategy and partnering of complementary skills represent a successful paradigm

\section{Outstanding Questions}

What are the mechanisms and underpinning genes that can be combined to increase yield potential and yield resilience reproducibly in a field environment?

Despite the multigenic nature of yield control, is it possible there are a few gene master regulators that could be targeted to increase crop yields - as in the Green Revolution which relied on improvement of traits underpinned by relatively few genes?

Is the regulation of sucrose use and allocation a major regulatory hub for yield and resilience to multiple stresses, and is thus an opportunity for yield improvement and for relieving sink limitations of photosynthesis by improving photosynthetic efficiency?

Can GM or genome-editing techniques be utilized for large improvements in yield potential and abiotic stress tolerance, or should other breeding and chemical methods be prioritized for these traits until the most promising gene targets in their cell and developmental contexts are identified and understood?

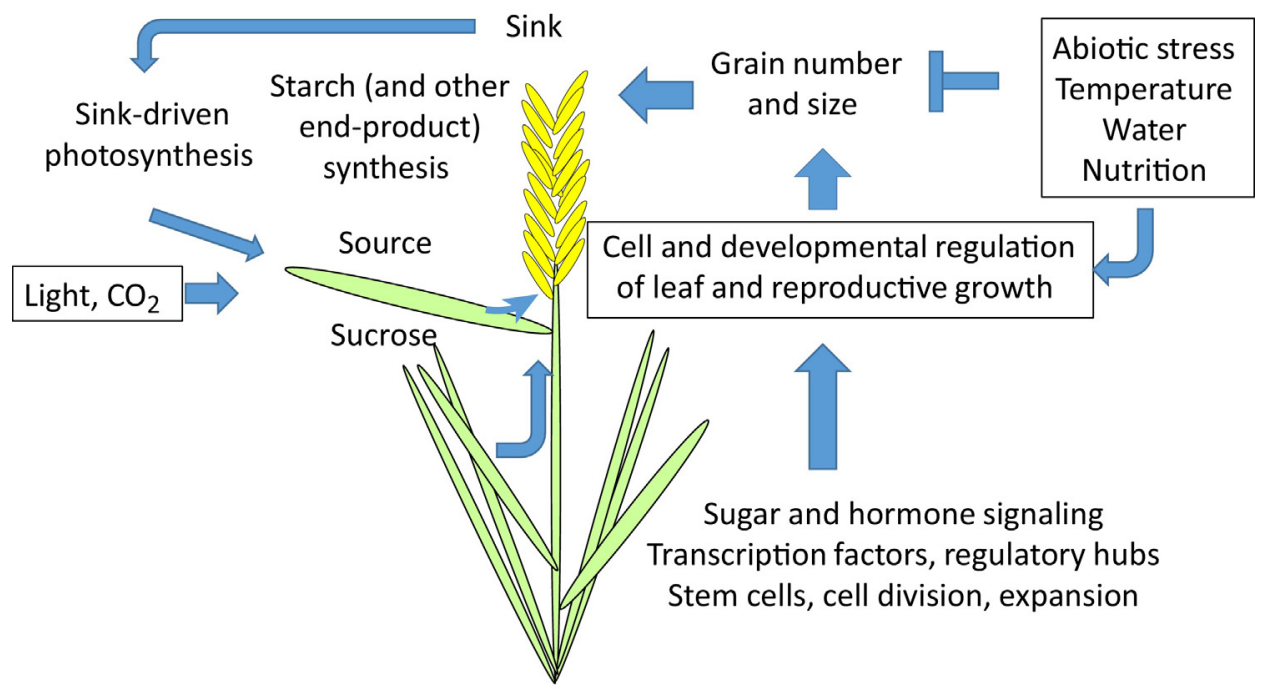

Trends in Plant Science

Figure 1. Combining Understanding of the Cell and Developmental Nature of Growth and Yield Control with Field Testing Is Necessary for the Successful Development of Genetically Modified (GM) and GenomeEdited Crops for Yield and Resilience. 
for how knowledge of fundamental science can benefit food security, and is particularly necessary if GM and future genome-editing approaches are to deliver on their promise of step-changes in yield in a range of environments (Figure 1, also see Outstanding Questions).

\section{Acknowledgments}

Rothamsted Research is strategically funded by the Biotechnology and Biological Sciences Research Council (BBSRC) of the UK. M.J.P. acknowledges support from the Designing Future Wheat Institute Strategic Programme and BBSRC grants BB/C51257X/1, BB/D006112/1 (SCIBS), and BB/N004205/1 (SARIC).

\section{Resources}

iwww.isaaa.org

iiwww.aphis.usda.gov/aphis/ourfocus/biotechnology/permits-notifications-petitions/petitions/petition-status

\section{References}

1. Chilton, M.D. et al. (1982) Agrobacterium rhizogenes inserts TDNA into the genomes of the host plant root cells. Nature 295 , 432-434

2. Fraley, R.T. et al. (1983) Expression of bacterial genes in plant cells. Proc. Natl. Acad. Sci. U. S. A. 80, 4803-4807

3. Klümper, W. and Qaim, M. (2014) A meta-analysis of the impacts of genetically modified crops. PLoS One 9, e111629

4. Brookes, G. and Barfoot, P. (2016) Global income and production impacts of using GM crop technology 1996-2014. GM Crops Food 7, 38-77

5. Gonsalves, D. et al. (1998) Transgenic virus-resistant papaya: a new hope for controlling papaya ringspot virus in Hawaii. APSnet Features http://dx.doi.org/10.1094/PHP-2000-0621-01-RV

6. Kamthan, A. et al. (2016) Genetically modified (GM) crops: milestones and new advances in crop improvement. Theor. Appl. Genet. 129, 1639-1655

7. Chawla, R. et al. (2012) Tuber-specific silencing of asparagine synthetase-1 reduces the acrylamide-forming potential of potatoes grown in the field without affecting tuber shape and yield. Plant Biotechnol. J. 8, 913-924

8. Lanahan, M.B. et al. Syngenta Participations AG. Self-processing plants and plant parts, US 7557262 B2

9. Richardson, T.H. et al. (2002) A novel, high performance enzyme for starch liquefaction discovery and optimisation of a low $\mathrm{pH}$ thermostable $\alpha$-amylase. J. Biol. Chem. 277, 26501-26507

10. Baucher, M. et al. (2003) Lignin: genetic engineering and impact on pulping. Crit. Rev. Biochem. Mol. Biol. 38, 305-350

11. Reddy, M.S.S. et al. (2005) Targeted down-regulation of cytochrome P450 enzymes for forage quality improvement in alfalfa (Medicago sativa L.). Proc. Natl. Acad. Sci. 102, 16573-16578

12. Suriyamongkol, P. et al. (2007) Biotechnological approaches for the production of polyhydroxyalkanoates in microorganisms and plants - a review. Biotechnol. Adv. 25, 148-175

13. Boehm, R. (2007) Bioproduction of therapeutic proteins in the 21 st century and the role of plants and plant cells as production platforms. Ann. N. Y. Acad. Sci. 1102, 121-134

14. Dunwell, J.M. (2000) Transgenic approaches to crop improvement. J. Exp. Bot. 51, 487-496

15. Albacete, A.A. et al. (2014) Hormonal and metabolic regulation of tomato fruit sink activity under salinity. J. Exp. Bot. 65, 60816095

16. Smidansky, E.D. et al. (2002) Enhanced ADP-glucose pyrophosphorylase activity in wheat endosperm increases seed yield. PNAS 99, 1724-1729

17. Hou, J. et al. (2014) Global selection on sucrose synthase haplotypes during a century of wheat breeding. Plant Physiol. 162, 1918-1929

18. Saalbach, I. et al. (2014) Increased grain yield and micronutrient concentration in transgenic winter wheat by ectopic expression of a barley sucrose transporter. J. Cereal Sci. 60, 75-81
19. Albecete, A.A. et al. (2014) Hormonal and metabolic regulation of source-sink relations under salinity and drought: from Plant survival to crop yield stability. Biotechnol. Adv. 32, 12-30

20. Lu, G. et al. (2015) OsPIN5b modulates rice (Oriza sativa) plant architecture and yield by changing auxin homeostasis, transport and distribution. Plant J. 83, 913-925

21. Liu, L. et al. (2015) Activation of Big Grain1 significantly improves grain size by regulating auxin transport in rice. PNAS 112, 1110211107

22. Rosenthal, D.M. et al. (2011) Overeexpressing the C3 photosynthesis cycle enzyme sedoheptulose-1-7-bisphosphatase improves photosynthetic carbon gain and yield under fully open air CO2 fumigation (FACE). BMC Plant Biol. 11, 123

23. Shani, Z. et al. (2004) Growth enhancement of transgenic poplar plants by overexpression of Arabidopsis thaliana endo-1,4- $\beta$-glucanase (cel1). Mol. Breed. 14, 321-330

24. Nuccio, M.L. et al. (2015) Plant trait gene expression cassette design. In Recent Advances in Gene Expression and Enabling Technology in Crop Plants (Azhakanandam, K., ed.), pp. 41-78, Springer

25. Preuss, S.B. et al. (2012) Expression of the Arabidopsis thaliana BBX32 gene in soybean increases grain yield. PLoS One 7 , e30717

26. Rice, E.A. et al. (2014) Expression of a truncated ATHB17 protein in maize increases ear weight at silking. PLoS One 9 , e94264

27. Jereme, L.M. et al. (2014) Stark comparative analysis of maize (Zea mays) crop performance: natural variation, incrementa improvements and economic impacts. Plant Biotechnol. J. 12, $941-950$

28. Zhang, Y.-C. et al. (2013) Overexpression of microRNA OsmiR397 improves rice yield by increasing grain size and promoting panicle branching. Nat. Biotechnol. 31, 848-852

29. Je, B.I. et al. (2016) Signaling from maize organ primordia via FASCIATED EAR3 regulates stem cell proliferation and yield traits. Nat. Genet. 48, 785-791

30. Lawlor, D.W. (2013) Genetic engineering to improve plant performance under drought: physiological evaluation of achievements, limitations, and possibilities. J. Exp. Bot. 64, 83-108

31. Shi, J. et al. (2015) Overexpression of ARGOS genes modifies plant sensitivity to ethylene, leading to improved drought tolerance in both Arabidopsis and maize. Plant Physiol. 169, 266-282

32. Rai, M.I. et al. (2015) The ARGOS gene family functions in a negative feedback loop to desensitize plants to ethylene. BMC Plant Biol. 24, 157

33. Guo, M. et al. (2014) Maize ARGOS1 (ZAR1) transgenic alleles increase hybrid maize yield. J. Exp. Bot. 65, 249-260

34. Shi, J. et al. (2017) ARGOS8 variants generated by CRISPR-Cas9 improve maize grain yield under field drought stress conditions. Plant Biotechnol. J. 15, 2017-2216 
35. Habben, J.E. et al. (2014) Transgenic alteration of ethylene biosynthesis increases grain yield in maize under field drought-stress conditions. Plant Biotechnol. J. 12, 685-693

36. Castiglioni, P. et al. (2008) Bacterial RNA chaperones confer abiotic stress tolerance in plants and improved grain yield in maize under water-limited conditions. Plant Physiol. 147, 446-455

37. Nemali, K.S. et al. (2015) Physiological responses related to increased grain yield under drought in the first biotechnologyderived drought-tolerant maize. Plant Cell Environ. 38, 1866-1880

38. Yang, S. et al. (2010) Narrowing down the targets: towards successful genetic engineering of drought-tolerant crops. Mol. Plant 3, 469-490

39. Skirycz, A. et al. (2011) Survival and growth of Arabidopsis plants given limited water are not equal. Nat. Biotechnol. 29, 212-214

40. Boyer, J.S. and Westgate, M.E. (2004) Grain yields with limited water. J. Exp. Bot. 55, 2385-2394

41. Boyer, J.S. (2010) Drought decision-making. J. Exp. Bot. 61, 3493-3497

42. Nuccio, M.L. et al. (2015) Expression of trehalose 6-phosphate phosphatase in maize ears improves yield in well-watered and drought conditions. Nat. Biotechnol. 33, 862-869
43. Goddijn, O.J.M. et al. (1997) Inhibition of trehalase activity enhances trehalose accumulation in transgenic plants. Plant Physiol. $113,181-190$

44. Paul, M.J. et al. (2001) Enhancing photosynthesis with sugar signals. Trends Plant Sci. 6, 197-200

45. Edmeades, G.O. (2013) Progress in Achieving and Delivering Drought Tolerance in Maize: An Update, ISAAA

46. Yadav, U.P. et al. (2014) The sucrose-trehalose 6-phosphate nexus: specificity and mechanisms of sucrose sensing by Tre6P. J. Exp. Bot. 65, 1051-1068

47. Zhang, Y. et al. (2009) Inhibition of Snf1-related protein kinase (SnRK1) activity and regulation of metabolic pathways by trehalose 6-phosphate. Plant Physiol. 149, 1860-1871

48. Pellny, T.K. et al. (2004) Genetic modification of photosynthesis with E. coli genes for trehalose synthesis. Plant Biotechnol. J. 2 , $71-82$

49. Schwachtje, J. et al. (2006) SNF1-related kinases allow plants to tolerate herbivory by allocating carbon to roots. Proc. Natt. Acad. Sci. U. S. A. 103, 12935-12940

50. Griffiths, C.A. et al. (2016) Chemical intervention in plant sugar signalling increases yield and resilience. Nature 540, 574-578

51. Kretzschmar, T. et al. (2015) A trehalose 6-phosphate phosphatase enhances anaerobic germination tolerance in rice. Nat Plants 1, 15124 